\title{
Synthesis, Characterization, and Modeling of Nanotube Materials with Variable Stiffness Tethers
}

\author{
S. J. V. Frankland ${ }^{1}$, M. N. Herzog ${ }^{2}$, G. M. Odegard ${ }^{1}$, T. S. Gates ${ }^{3}$, C. C. Fay ${ }^{3}$ \\ ${ }^{1}$ National Institute of Aerospace, Hampton, VA \\ ${ }^{2}$ National Research Council, Hampton, VA \\ ${ }^{3}$ NASA Langley Research Center, Hampton, VA
}

\begin{abstract}
Synthesis, mechanical testing, and modeling have been performed for carbon nanotube based materials. Tests using nanoindentation indicated a six-fold enhancement in the storage modulus when comparing the base material (no nanotubes) to the composite that contained $5.3 \mathrm{wt} \%$ of nanotubes. To understand how crosslinking the nanotubes may further alter the stiffness, a model of the system was constructed using nanotubes crosslinked with a variable stiffness tether (VST). The model predicted that for a composite with $5 \mathrm{wt} \%$ nanotubes at random orientations, crosslinked with the VST, the bulk Young's modulus was reduced by 30\% compared to the noncrosslinked equivalent.
\end{abstract}

\section{INTRODUCTION}

Nanostructured composites are of importance to the aerospace industry as candidate materials for future light-weight structural components. Interest continues to center on the suitability of carbon nanotube based composites for structural applications. However, the theoretical improvements to mechanical performance have been difficult to realize because of limited ability to disperse carbon nanotubes in polymeric materials, uncontrolled nanotube alignment in the composites, and weak polymer-carbon nanotube interfaces within the material. Crosslinked nanotube materials provide some potential solutions to these problems in that the nanotube/crosslinker interface should be strengthened by the presence of the additional covalent linkages and that the crosslinking agent would intercalate between the nanotubes resulting in better dispersion. Previous work has proposed the synthesis of crosslinked nanotube materials with short rigid tethers and has used multi-scale model to address the effect of crosslinking the carbon nanotubes on the constitutive properties [1]. In related work, others have fabricated polyacrylonitrile (PAN) fibers reinforced with carbon nanotubes that demonstrated tensile modulus increases of up to $100 \%$ at $10 \mathrm{wt} \%$ single-walled carbon nanotube (SWNT) content [2] and $30 \%$ increases in modulus at $5 \mathrm{wt} \%$ multi-walled nanotube content [3]. In the SWNT/PAN fibers, the nanotubes also align well and are partially exfoliated [2].

In the present work, the influence of crosslinking is explored with a multi-scale model. The materials of interest consist of SWNT crosslinked with a long variable stiffness tether (VST). The VST crosslinking agent (Figure 1) consists of rigid and flexible sections which are capable of forming covalent linkages to and packing between the SWNT. The objective of the work is to demonstrate the effect of nanotube functionalization on the constitutive properties of the crosslinked nanotube material. To help guide the model, baseline material (no nanotubes) and a SWNT composite with VST were fabricated and tested. The degree of crosslinking in the synthesized material is unknown. To explore the relative influence of crosslinking on mechanical performance, the multi-scale model incorporates the chemical specificity of the VST and the 
covalent linkages to SWNT. Representative volume elements (RVE) of portions of the molecular structure anticipated in the materials are developed with molecular dynamics (MD) simulation. Equivalent continuum modeling is then used in combination with the MD simulations to determine the constitutive properties of the RVEs as transversely isotropic solids. Finally, the Mori-Tanaka micromechanics method is used to determine the Young's modulus of the overall crosslinked nanotube material. The paper will first present the details of the synthesis and experimental methods, and then describe the multi-scale model and results.

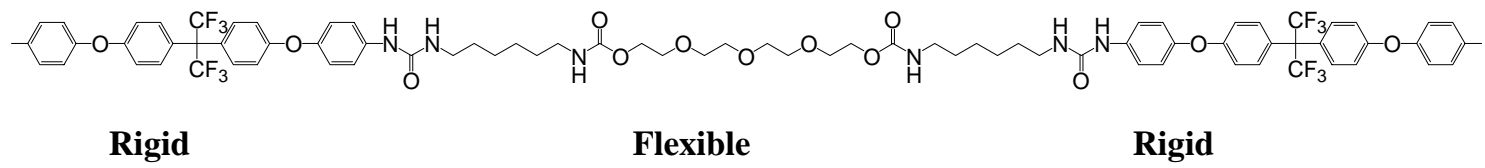

Figure 1. Chemical structure of the variable stiffness crosslinking agent: VST.

\section{MATERIAL SYNTHESIS AND EXPERIMENTAL CHARACTERIZATION}

The crosslinked nanotube materials are synthesized via reaction of the amine terminated VST (Figure 1) and SWNTs using the method of Tour, et al. [4]. The SWNTs were purchased from NanoTechLabs Inc. of Clemson, SC and purified according to literature procedures [5]. The VST crosslinking agent was synthesized from 1,6-diisocyanatohexane, tetraethyleneglycol, and 2,2bis[4-(4-aminophenoxy)phenyl] hexafluoropropane (dried) by a procedure that will be presented elsewhere. Its chemical structure was verified with ${ }^{1} \mathrm{H}-\mathrm{NMR}$ and FT-IR. All the synthetic manipulations were performed using standard Schlenk techniques unless otherwise noted. To form the VST/SWNT crosslinked material, SWNTs, VST, and dichlorobenzene were briefly sonicated. Isoamyl nitrite was then added, and the reaction was heated to $75^{\circ} \mathrm{C}$ for 4 hours. The resulting black solid was washed sequentially with pentane, diethyl ether, acetone, and then pentane again. The black solid was then dried to constant weight at $180^{\circ} \mathrm{C}$ under vacuum to yield a black powder. The residue remaining from thermogravimetric analysis in argon at $600^{\circ} \mathrm{C}$ was 5.3 wt \% which provides a lower limit on the nanotube weight fraction in the material [6]. Functionalization of the carbon nanotubes in VST/SWNT sample was verified by Raman spectroscopy. At an excitation wavelength of $785 \mathrm{~nm}$, the ratio of the $1585-1589 \mathrm{~cm}^{-1}$ band to the $1289-1290 \mathrm{~cm}^{-1}$ band was $1: 1$. (For pressed non-functionalized SWNTs from the same source, the ratio was 2:1.)

The VST (baseline) and SWNT/VST (composite) materials were then mechanically tested at room temperature using dynamic nanoindentation. Disks of the materials were made using thermal compression molding. An ungreased $0.8 \mathrm{~cm}$ circular bored mold was charged with approximately $200 \mathrm{mg}$ of material. The sample was cold-pressed at 15,000 psi for $5 \mathrm{~min}$. The sample was then transferred to a hot press and pressed at $20^{\circ} \mathrm{C}$ above the samples $\mathrm{T}_{\mathrm{g}}$ at $400 \mathrm{psi}$ for $1 \mathrm{~h}$. The sample was then allowed to cool to $50{ }^{\circ} \mathrm{C}$ while maintaining pressure. A surface of the disk was then polished in preparation for nanoindentation. The room temperature storage modulus was measured using dynamic nanoindentation and the procedures outlined in Ref. [7]. Multiple indentations were performed on each sample for a depth, strain rate, harmonic displacement, and harmonic frequency of $1500 \mathrm{~nm}, 0.05 \mathrm{~s}^{-1}, 10 \mathrm{~nm}$, and $50 \mathrm{~Hz}$, respectively. The storage modulus values were averaged over the depth range of 200-1400 nm, and the uncertainty values were the standard deviation of the mean based on repeat tests of the same sample. 


\section{MODELING AND SIMULATION}

A multi-scale method was employed to determine the mechanical properties of the crosslinked composite materials. This method involved four stages and has been described for this system elsewhere [8]. A brief summary of the method will be given here.

First, molecular force fields were chosen to describe the contributions to the chemical potential energy of system components. Standard parameters were used here, but some modification was required to account for the chemical specificity of the VST crosslinking agent. The crosslinking nanotube material was composed of SWNTs and the VST crosslinking material. The AMBER force field was used for the SWNT [9]. Non-bonded van der Waals interactions between carbon atoms participating in 1-4 dihedral angles were included as scaled by a factor of 0.5 for Lennard-Jones interactions and 0.83 for electrostatic interactions. Standard AMBER parameters are used for the VST crosslinking agent to describe the bond stretches, angles, dihedrals, improper dihedrals, and Lennard-Jones interactions. However, the bond lengths, angles, and partial atomic charges were derived from $a b$ initio calculations peformed using NWChem [10]. The molecular geometry of the VST was optimized using a Hartree-Fock calculation with a STO-3G basis set. The partial atomic charges were subsequently chosen from an ESP fit to a Hartree-Fock with a 6-31G* basis set at the optimized molecular geometry. Some assumptions were made and are described in [8]. Except for the covalent bonds added between VST and the SWNT, the interactions between the two were modeled with standard Lennard-Jones parameters.

Second, RVEs were generated for the material using MD simulations performed using DLPOLY [11]. The RVEs implemented here were parallelepiped elements which have periodic boundary conditions in all three dimensions. To establish a range of materials based on the degree of crosslinking, four different RVEs were developed. The first RVE (denoted Bundle) was a non-crosslinked SWNT bundle of 9 nanotubes of length $5.4 \mathrm{~nm}$. The second and third RVEs had 0.45\% (Material A) and $1.8 \%$ (Material B) of the nanotube carbon atoms covalently bonded to VST crosslinking agent respectively. Material A also had $1 / 4$ the number of VST molecules of Material B. The fourth RVE (Material B (unreacted)) had the same number of VST molecules as in Material B, but no covalent bonds to the nanotubes. Instead, the VST molecules in $\mathbf{B}$ (unreacted) were terminated with hydrogen. The general or idealized structure which was periodic in three dimensions is shown in Figure 2(a). From the idealized structure, a compression algorithm was used to form the equilibrium structure (Figure 2(b)) at $300 \mathrm{~K}$. The final simulation box dimensions were chosen from an NPT simulation at 1 atm and $300 \mathrm{~K}$. These simulations were carried out for 50,000 steps with a time step of 0.5 fs.

(a)

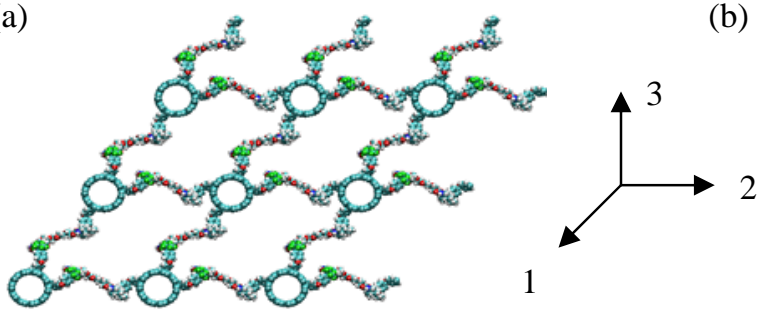

(b)

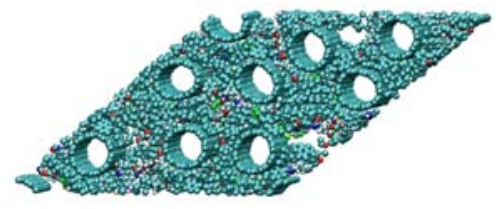

Figure 2. (a) Idealized and (b) equilibrium RVEs of VST/SWNT material.

Third, MD simulation was used in conjunction with the equivalent continuum method to determine the elastic constants of the RVEs. The energies of deformation of the molecular 
model were calculated from the MD simulations and equated to the strain energy of an equivalent-continuum solid, for identical sets of boundary conditions. Each boundary condition was used to calculate a single parameter in the assumed constitutive relationship for the equivalent continuum. These simulations were performed for 100,000 steps at $0.5 \mathrm{fs}$ each, at a strain rate of 0.0025 per 10,000 steps. From the molecular structure shown in Figure 2, it was assumed that the RVE had transversely isotropic symmetry. Therefore, the constitutive equation for the RVE model was

$$
\begin{array}{cc}
\sigma_{11}=C_{11} \varepsilon_{11}+C_{12} \varepsilon_{22}+C_{12} \varepsilon_{33} & \sigma_{23}=(1 / 2)\left(C_{22}-C_{23}\right) \gamma_{23} \\
\sigma_{22}=C_{12} \varepsilon_{11}+C_{22} \varepsilon_{22}+C_{23} \varepsilon_{33} & \sigma_{13}=G_{12} \gamma_{13} \\
\sigma_{33}=C_{12} \varepsilon_{11}+C_{23} \varepsilon_{22}+C_{22} \varepsilon_{33} & \sigma_{12}=G_{12} \gamma_{12}
\end{array}
$$

where $\sigma_{i j}$ and $\varepsilon_{i j}$ are the stress and strain components, respectively $(i, j=1,2,3)$, and $C_{i j}$ and $G_{i j}$ are the elastic stiffness components of the equivalent continuum, where the usual contracted notation is used. The subscripts refer to coordinate directions with the 1-axis along the nanotube, the 2, 3axes transverse to the nanotube (Figure 2). Because five independent elastic constants are required to complete the constitutive equation, five independent boundary conditions were applied to the molecular and equivalent-continuum models.

In the final stage of the model, the RVE was assumed to represent an "effective fiber" incorporating both nanotube and surrounding VST material. It was assumed that the effective fibers of the VST/SWNT material were embedded in the VST matrix with random orientation. The bulk stiffness components of the resultant composite material were determined using the Mori-Tanaka micromechanics method [12]. The effective fibers were cylindrical with an aspect ratio of 1:1000. The composite matrix had a Young's modulus of $0.57 \mathrm{MPa}$, assumed equal to the storage modulus of the VST reported from nanoindentation, and a Poisson's ratio of 0.4. Perfect bonding between the effective fiber and the matrix was assumed. The random orientation of the effective fibers in three dimensions was incorporated into the calculation of the composite stiffness using the following function on the stiffness components

$$
\left\langle C_{i j m n}\right\rangle=\left(\kappa-\frac{2}{3} \mu\right)\left(\delta_{i j} \delta_{m n}\right)+\mu\left(\delta_{i m} \delta_{j n}+\delta_{i n} \delta_{j m}\right)
$$

where $i, j, m, n=1,2,3$; the expanded notation and indicial summation conventions are used, $\delta_{i j}$ is the Kronecker delta; and

$$
\kappa=\frac{1}{9} C_{i i j j} \quad \mu=\frac{1}{10}\left(C_{i j i j}-\frac{1}{3} C_{i i j j}\right)
$$

Therefore, the angle-bracketed terms in Eq. (3) are isotropic, resulting in an isotropic material and a single Young's modulus for the bulk composite.

\section{RESULTS AND DISCUSSION}

The storage moduli for the VST and SWNT/VST materials synthesized were measured by nanoindentation. For the VST material (baseline), the storage modulus was $0.56 \pm 0.05 \mathrm{GPa}$, and for the VST/SWNT composite material, it was $2.9 \pm 0.02 \mathrm{GPa}$. The nanotube weight fraction was measured using TGA to be $5.3 \mathrm{wt} \%$.

At first, considering only the transversely isotropic VST/SWNT RVE, the elastic constants $\mathrm{C}_{11}$ (longitudinal) and $\mathrm{G}_{12}$ (in-plane shear) are plotted in Figure 3 as a function of the nanotube volume fraction in the RVE. As crosslinking agent was added in the simulations the nanotubes 
separated (Figure 2(b)). Figure 3(a) indicates that the constant $\mathrm{C}_{11}$ had a linear dependence on nanotube volume fraction. Likewise, the bulk shear modulus $\mathrm{K}_{12}$ exhibited similar behavior (not shown). The calculated value of $\mathrm{G}_{12}$, Figure 3(b), increased by more than sixfold for Material $\mathbf{B}$ relative to the nanotube bundle even though the nanotube volume fraction in this RVE was three times lower than the nanotube volume fraction in the bundle. The effect of the crosslinking alone shows up in the four fold increase in $G_{12}$ when comparing materials $\mathbf{B}$ and $\mathbf{B}$ (unreacted) at approximately the same nanotube volume fraction. The bulk shear modulus for the RVE, $\mathrm{K}_{23}$, was comparable for all three VST/SWNT materials, but was less than $90 \%$ of the $\mathrm{K}_{23}$ of the SWNT bundle RVE. The transverse shear modulus, $\mathrm{G}_{23}$, was independent of nanotube volume fraction in the VST/SWNT materials.

(a)

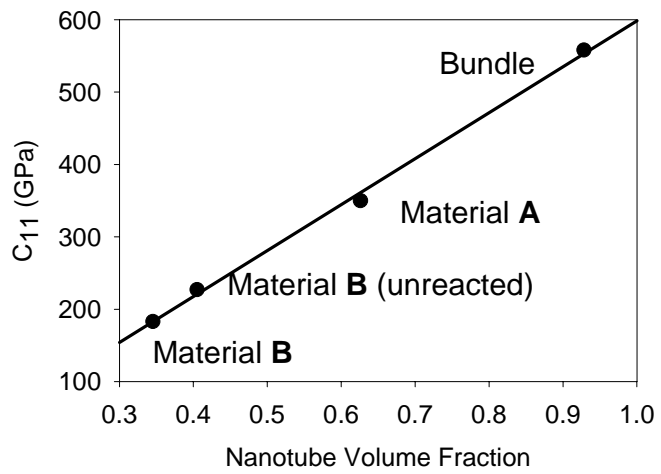

(b)

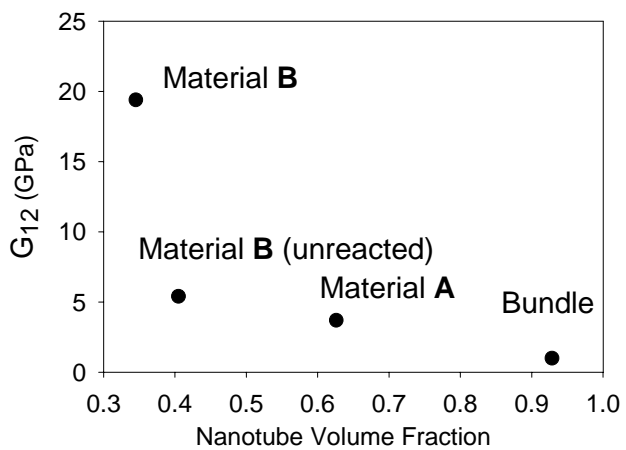

Figure 3. Elastic constants (a) $\mathrm{C}_{11}$ and (b) $\mathrm{G}_{12}$ of the molecular RVEs.

In Figure 4, the isotropic composite Young's modulus is plotted as a function of nanotube weight fraction for the randomized composites made from each of the four effective fiber RVEs embedded in the VST matrix material. It was assumed that the VST density was $1 \mathrm{~g} / \mathrm{cm}^{3}$. The nanotube bundle and the non-bonded VST/SWNT composite behaved similarly despite the threefold difference in nanotube volume fraction content per RVE. At all nanotube weight fractions, the composite Young's modulus decreased with increasing nanotube functionalization. At the 5 $\%$ nanotube weight fraction, the Young's modulus of Material A was reduced to $70 \%$ of the Young's modulus of the SWNT bundle material, while Material B was $30 \%$ that of SWNT bundle material. The exact cause of the decrease predicted by the Mori-Tanaka method is as yet unknown, but seems to be related to the increasing values of $G_{12}$ given in Figure 3(b). It is doubtful that the change in $\mathrm{C}_{11}$ values (Figure 3(a)) was responsible for this decrease in composite modulus due to the fact that the non-bonded material and the SWNT bundle had very different $C_{11}$ values (Figure 3(a)), but they both yield similar overall Young's moduli (Figure 4).

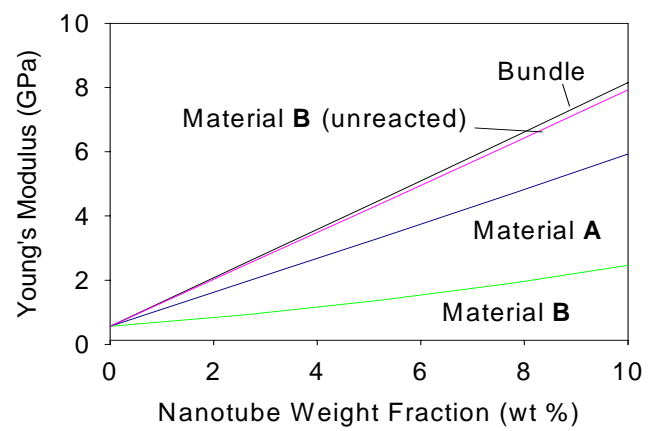

Figure 4. Young’s modulus of crosslinked nanotube materials from multi-scale model. 
The synthesized composite material, which had a nanotube weight fraction of $5.3 \%$, had a measured storage modulus that fell between the range of predicted Young's modulus for Materials A and $\mathbf{B}$ (Figure 4). While this comparison warrants further study, it is confirmation that the model and the experiment are within reasonable range of one another.

\section{CONCLUSIONS}

Carbon nanotube materials crosslinked with a variable stiffness molecular tether (VST) have been modeled with a multi-scale method. At the atomistic scale, the molecular representative volume elements demonstrate that the axial stiffness $\left(C_{11}\right)$ is more dependent on the nanotube volume fraction than the degree of crosslinking. The in-plane shear modulus of the RVE $\left(\mathrm{G}_{12}\right)$ is found to increase as degree of crosslinking is increased. At the continuum scale, the isotropic composite Young's modulus decreases with increasing nanotube functionalization. Specifically, a material with $5 \mathrm{wt} \%$ nanotubes and 1.8\% degree of crosslinking, has a Young's modulus that is $30 \%$ less that of the non-crosslinked material. Parametric studies such as those performed herein point to the relative sensitivity of the material stiffness to the degree of functionalization.

\section{ACKNOWLEDGMENTS}

S. J. V. Frankland and G. M. Odegard gratefully acknowledge support from the NASA Cooperative Agreement with the National Institute of Aerospace. This research was performed while M. N. Herzog held a National Research Council Research Associateship Award at NASA Langley Research Center.

\section{REFERENCES}

1. S. J. V. Frankland, M. N. Herzog, G. M. Odegard, T. S. Gates, and C. C. Fay, 'Modeling and Characterization of Elastic Constants of Functionalized Nanotube Materials,' Mat. Res. Soc. Symp. Proc. 791 (2003).

2. T. V. Sreekumar, T. Liu, B. G. Min, H. Guo, S. Kumar, R. H. Hauge, and R. E. Smalley, Adv. Materials, 16, 58 (2004).

3. M. C. Weisenberger, E. A. Grulke, D. Jacques, T. Rantrell, and R. Andrews, J. Nanoscience and Nanotechnology, 3, 535 (2003).

4. J. L. Bahr and J. M. Tour, Chem. Mater. 13, 3823 (2001).

5. I. W. Chiang, B. E. Brinson, A. Y. Huang, P. A. Willis, M. J. Bronikowski, J. L. Margrave, R. E. Smalley, and R. H. Hauge, J. Phys. Chem. B 105, 8297 ( 2001).

6. J. L. Bahr, J. Yang, D. V. Kosynkin, M. J. Bronikowski, R. E. Smalley, and J. M Tour, J. Am. Chem. Soc. 123, 6536 (2001).

7. G.M. Odegard, T. Bandorawalla, H.M. Herring, and T.S. Gates, Proc. of the 2003 SEM Annual Conference and Exposition on Experimental and Applied Mechanics, June 2-4, 2003, Charlotte, NC.

8. S. J. V. Frankland, G. M. Odegard, M. N. Herzog, T.S. Gates, and C.C. Fay, Proc.of the ASC/ASTM-D30 Joint 19th Annual Technical Conference, Atlanta, GA, Oct 17-20, 2004.

9. W.D. Cornell, P. Cieplak, C.I. Bayly, I.R. Gould, K.M. Merz, D.M. Ferguson, D.C. Spellmeyer, T. Fox, J.W. Caldwell and P.A. Kollman, J. Am. Chem. Soc. 117, 5179 (1995).

10. R. J. Harrison, et al. "NWChem, A Computational Chemistry Package for Parallel Computers, Version 4.1," Pacific Northwest National Laboratory, Richland, Washington.

11. W. Smith, and T. R. Forester. DL-POLY, Warrington, England: The Council for the Central Laboratory of the Research Councils, 1996.

12. T. Mori and K. Tanaka, Acta Metallurgica, 21, 571 (1973). 